\title{
Compliance with social norms as an evolutionary stable equilibrium
}

Francisco Cabo and Ana García-González and Mercedes Molpeceres-Abella

\begin{abstract}
This paper analyzes the compliance with social norms optimally established by a benevolent central planner. Since compliance is costly, agents have an incentive to free-ride on others, in a public good game. We distinguish two types of agents: standard pro-self agents (Sanchos) whose payoffs are defined by a prisoner's dilemma game dominated by the non-compliance strategy, and pro-social Quixotes, who still have an incentive to free-ride, although prefer compliance over mutual defection (as in a snowdrift game). Compliance is analyzed in a two-population evolutionary game considering an imitative revision protocol. Individuals from one population play against and imitate agents within their own but also the other population. Inter-population interaction and imitation allow us to investigate under which circumstances some Sanchos might imitate compliant Quixotes, so escaping the non-compliance equilibrium characteristic of an isolated population of Sanchos. Correspondingly, we analyze the conditions under which the interaction with the population of selfish Sanchos increases or decreases the compliance rate among altruistic Quixotes.
\end{abstract}

Francisco Cabo

IMUVa, Universidad de Valladolid, Avda Valle Esgueva,6 e-mail: pcabo@eco.uva.es

Ana García-González

IMUVa, Universidad de Valladolid, Avda Valle Esgueva,6 e-mail: anagar@eco.uva.es

Mercedes Molpeceres-Abella

Universidad de Valladolid, Avda Valle Esgueva,6 e-mail: abella@eco.uva.es 
Keywords: Two-population evolutionary game, heterogeneous preferences, prisoner's dilemma game, snowdrift game, social norms.

\section{Introduction}

Compliance with social norms can be analyzed as a collective action problem. Although collective actions may be jointly beneficial, a classical result, as stated by Olson (1971), is that no self-interested person would contribute in the production of a public good (except in the case of a very small group or under a mechanism like coercion). This zero contribution thesis corresponds to the Nash equilibrium in a prisoner's dilemma game. For the public good game (the generalization of the prisoner's dilemma to a large number of players), defection is the dominant strategy. Thus, classical game theory and also evolutionary game theory predict zero contribution (see, for example, Miller and Andreoni 1991). Nevertheless, experimental economics seems to contradict this zero contribution hypothesis. For example, Andreoni (1988) and later Ostrom (2000), revise the literature on public goods experiments, finding positive levels of contribution in one-shot and repeated games. Many examples of collective actions also exist in everyday life: voting, not cheating on taxes, contributions to voluntary associations, or recycling.

The literature provides alternative explanations to reconcile the standard theory with the evidence of individual behavior. Andreoni (1988) suggests that individual behavior, at least in the laboratory, is not exclusively oriented by self-interest but also by factors like altruism, social norms or bounded rationality. Here, we focus on the idea that individuals, or at least some individuals, can derive enjoyment from collaboration independently of how much their actions benefit others. This effect is called intrinsic satisfaction in de Young (1996), or "warm-glow" in the more standard terminology, like in Andreoni (1990). As long as social norms are perceived as ideal forms of behavior, compliance provides a warm-glow to each particular compliant agent, which is a supplementary benefit to the public good. ${ }^{1}$ Alternatively,

\footnotetext{
${ }^{1}$ Other factors not analyzed here are inequity aversion, reciprocal altruism, sense of identity or preference for efficiency (see, Alger and Weibull 2013 and references therein). The temporal di-
} 
individuals get dis-utility from doing bad. Thus, as long as not following social norms is perceived as doing bad, defection involves a cold-prickle, following the terminology in Andreoni (1995), who highlighted the asymmetry between doing good versus not doing bad.

An economy populated by different types of individuals, some more inclined to collaborate than others was proposed by Ostrom (2000), from an evolutionary perspective. The existence of "norm-using" individuals, unlike the standard rational egoists, helps bring the theory closer to the findings in the laboratory. ${ }^{2}$ More recently, Grafton et at. (2017), consider the society as a mixture of standard "Nashian" individuals (who maximize their utility taking as given the strategies of others) and "Kantian" individuals (who do not do anything that they would not like if everyone behaves in the same way). We consider a society composed of two different types of agents: rational egoists or pro-self individuals, which we call Sanchos; and normusing or pro-social individuals, called Quixotes. Both follow a "Nashian" behavior, and their strategic behavior changes according to the evolutionary dynamics.

The preferences of rational egoist Sanchos are captured by a payoff matrix of the prisoner's dilemma type with a strong incentive to free-ride (the most widely used paradigm to study human cooperation). In contrast, pro-social ${ }^{3}$ Quixotes experience a warm-glow from compliance, together with a cold-prickle from defection. The free-riding incentive still persists when the opponent complies, however, if the opponent defects, compliance is their best strategy. Thus, for pro-social Quixotes the compliance dilemma is relaxed, described as a snowdrift game (also known as hawk-dove or chicken game), as proposed, for example, in Doebeli et al. (2004). Our

mension can also make compliance more rewarding. For pollution problems, current abatement activities can be beneficial when the future cleaner environment is taken into account. An interesting example is Breton et al. (2010), who propose a dynamic game for international environmental agreements that takes into account the pollution stock dynamics, as well as the membership dynamics, defined by an evolutionary process.

2 The mixture of rational egoists and norm-users results from an indirect evolutionary approach, proposed by Güth and Yaari (1992) and Güth (1995). Preferences are also considered as strategies subject to evolutionary selection in Alger and Weibull (2013), for whom natural selection has built preferences as a convex combination between selfishness and morality.

${ }^{3}$ In the same way as Don Quixote sought to behave in accordance with his reading of chivalry, pro-social individuals perceive social norms as ideal forms of behavior. 
setting follows Cabo and García-González (2018) who analyze a two-population evolutionary game in between a symmetric and an asymmetric game. Individuals belonging to one population play against and imitate individuals within their own and also the other population. The evolutionary dynamics converges towards a mixed strategy equilibrium at which some Sanchos might find it attractive to imitate the compliant behavior of Quixotes, though it is not in their best interest. This answers the question which typically intrigues a reader of "The Ingenious Gentleman Don Quixote of La Mancha": ${ }^{4}$ What led the pragmatic and rational Sancho to follow the "madness" of the morally oriented Don Quixote? Or put differently: under which circumstances does the existence of a population of pro-social individuals induce standard pro-self individuals to comply with the social rules when it is in their best interest not to do so? A positive answer requires a much higher reward to compliance for Quixotes than for Sanchos.

We extend Cabo and García-González (2018) by relaxing the assumption that the global population is equally divided between Sanchos and Quixotes. We analyze how compliance decisions in either population are affected by their relative sizes. Considering a pairwise imitation protocol, the relative size of each population determines the likelihood of being paired with an individual within this particular population. At the same time, it directly influences the share of compliance in the global population and, in consequence, the incentives to free-ride when others comply or to comply when others do not.

The analysis shows that the likelihood that some Sanchos imitate the compliant behavior of Quixotes increases with the ratio of Sanchos in the overall population. ${ }^{5}$ Conversely, when the ratio of Sanchos is very small, they would certainly freeride on compliant Quixotes, unless these latter get a very large warm-glow from compliance. This warm-glow makes it more likely compliance among Sanchos, and it also increases the actual compliance rates for both agents, Sanchos and Quixotes.

A larger ratio of Sanchos undoubtedly reduces the share of compliance in the global population. However, its effect on the share of compliance within the popula-

\footnotetext{
${ }^{4}$ Originally titled: El ingenioso hidalgo don Quijote de la Mancha, by Miguel de Cervantes Saavedra (1605 Part I and 1615 Part II).

${ }^{5}$ Similar result is obtained in Bontems and Rotillon (2000), for pollution compliance in a population divided between honest polluters (always comply) and opportunistic individuals.
} 
tion of Quixotes is twofold. A snowdrift effect induces Quixotes to increase compliance because the compliance rate in the global population is smaller. Conversely, an imitation effect leads Quixotes to reduce compliance following the non-compliant behavior of Sanchos. The positive effect becomes more important the greater the warm-glow, making more likely that a higher ratio of Sanchos induces higher compliance among Quixotes. On the other hand, the ratio of Sanchos, first raises compliance within this population although, with less and less Quixotes, Sanchos hardly meet and imitate Quixotes and compliance rates decrease to zero.

The rest of the paper is organized as follows. Section 2 presents the preferences of Sanchos and Quixotes. The two-population evolutionary game and the associated dynamics are introduced in Section 3. The two types of equilibria with and without compliance among Sanchos are computed in Section 4. Section 5 studies how compliance in each population is affected by the discrepancy between Sanchos and Quixotes, and by the relative sizes of these populations. It also presents some real life examples. Section 6 concludes.

\section{Two distinct populations}

This section explains the distinct behavior of pro-self Sanchos and norm-using Quixotes when they face a collective action problem like compliance with social norms. We assume that if the social norms are followed by all individuals, then they all will be better off. ${ }^{6}$ The collective action problem is perceived as: a prisoner's dilemma game for Sanchos, and a snowdrift game for Quixotes.

The population of Sanchos represents the standard rational agents, whose payoff matrix collects the canonical representation of a collective action problem:

\begin{tabular}{c|c|c} 
& $\mathrm{C}$ & $\mathrm{D}$ \\
\hline $\mathrm{C}$ & $(1,1)$ & $(1-d, 1+c-d)$ \\
$\mathrm{D}$ & $(1+c-d, 1-d)$ & $(0,0)$ \\
\hline
\end{tabular}

\footnotetext{
${ }^{6}$ Norms are optimally chosen by a benevolent central planner, or they are the result of a social learning process.
} 
Net gains when both players comply are normalized to 1 . Parameter $c$ represents the effort or the cost of compliance, and $d$ is the damage caused by a non-compliant player. Thus, the gap $c-d>0$ determines the incentive to free ride on the other, which induces the agent to disobey as long as his opponent complies. Moreover, in a prisoner's dilemma game a player prefers defection also when the opponent defects. This incentive is measured by $1-d<0$, which is less pronounced than the free-riding incentive if the opponent complies:

$$
\sigma \equiv c-d-(d-1)>0, \quad \text { with } \quad 1<d<c .
$$

Notice that the restriction $1<d<c$ ensures that defection is the dominant strategy among Sanchos. The restriction $\sigma>0$ is that the sum of the off-diagonal payoffs is greater than the sum of the diagonal payoffs. Thus, in the population of Sanchos the player's gains from defection, as opposed to compliance, is greater when the opponent complies, i.e. $c-d>d-1$. Under this prisoner's dilemma specification, the unique Nash equilibrium is the standard situation of mutual defection.

The population of Quixotes attains a greater reward for compliance and a lower reward for defection than the population of Sanchos. They attach a warm-glow to compliance, $w g \geq 0$, and a cold-prickle to defection, $c p \geq 0$. Thus, their payoffs can be computed as:

\begin{tabular}{c|c|c} 
& $\mathrm{C}$ & $\mathrm{D}$ \\
\hline $\mathrm{C}$ & $(1+w g, 1+w g)$ & $(1-d+w g, 1+c-d-c p)$ \\
$\mathrm{D}$ & $(1+c-d-c p, 1-d+w g)$ & $(-c p,-c p)$ \\
\hline
\end{tabular}

The warm-glow associated with compliance and the cold-prickle associated with defection makes compliance more attractive and defection less so. The social dilemma for the population of Quixotes is described as a snowdrift (Hawk-Dove or chicken) game. This implies that the joint effect of the warm-glow and the cold-prickle is not enough to counterbalance the free-riding incentive when the opponent complies: $w g+c p<c-d$. In contrast, this joint effect is strong enough to induce compliance when the opponent disobeys $w g+c p>d-1$. In what follows, this joint effect will be denoted as $\varepsilon=w g+c p$. In consequence, the warm-glow from compliance and the cold-prickle from defection can be expressed as: $w g=\alpha \varepsilon, c p=(1-\alpha) \varepsilon$, with $\alpha \in[0,1]$. While $\varepsilon$ is a quantitative measure of the distance between Sanchos and 
Quixotes, parameter $\alpha$ can be interpreted as a qualitative measure of this distance, that measures the relative importance of the warm-glow over the cold-prickle. Note that $w g / c p=\alpha /(1-\alpha)$, and it moves between 0 and $\infty$ as $\alpha$ moves between 0 and 1. Given this notation, the snowdrift structure of this matrix requires conditions in (1) together with:

$$
d-1<\varepsilon<c-d .
$$

For such a game, mutual defection is no longer a Nash equilibrium. It is characterized by a Nash equilibrium in mixed strategies: ${ }^{7}$

$$
(\Delta, 1-\Delta)=\left(\frac{\varepsilon-(d-1)}{\sigma}, \frac{c-d-\varepsilon}{\sigma}\right) \in(0,1) \times(0,1) .
$$

The expression $\Delta$ represents a relative measure of the the incentive to comply when the opponent disobeys. Corresponding, $1-\Delta$ provides the relative measure of the free-riding incentive when the opponent complies. These two values are positive under condition (2) and the assumption $\sigma>0$. The greater the absolute distance which separates Sanchos and Quixotes, $\varepsilon$, the greater the incentive to comply if the opponent disobeys, and the lower the free-riding incentive if the opponent complies (see Figure 1). However, $\Delta$, is independent of $\alpha$, i.e. of whether Quixotes attach a large warm-glow to compliance or a strong cold-prickle to non-compliance.

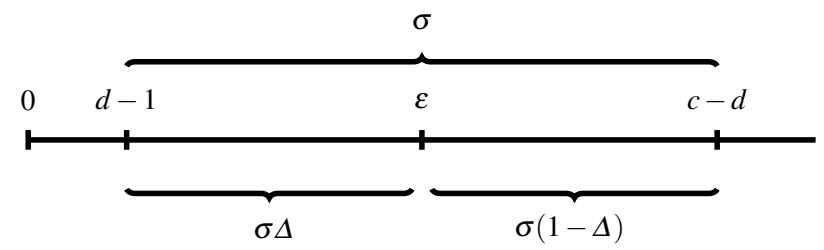

Fig. 1 Incentives to comply/free-ride

In addition to the definition of $\Delta$, we introduce two new expressions:

$$
\Delta_{w g}=\frac{\alpha \varepsilon-(d-1)}{\sigma}, \quad \Delta_{c p}=\frac{(1-\alpha) \varepsilon-(d-1)}{\sigma} .
$$

These expressions can be positive or negative. A positive $\Delta_{w g}$ implies that the warmglow from compliance for Quixotes, $\alpha \varepsilon$, is by itself, a strong enough incentive to

\footnotetext{
${ }^{7}$ As well as two asymmetric pure Nash equilibria, $(1,0),(0,1)$.
} 
induce compliance when the opponent defeats. Likewise, a positive $\Delta_{c p}>0$ implies that the Quixote's preference for compliance when the opponent defeats could be based only on the cold-prickle she associates to defection (with no need of warmglow from compliance).

In what follows, unless said otherwise, the illustrative examples will consider the following parameters' values:

$$
c=1.6, d=1.1, \varepsilon=0.4 \text {. }
$$

For these parameters it holds that $\sigma=0.4>0, \Delta=0.75, d-1=0.1<c-d=0.5$, satisfying conditions (1) and (2). Moreover, $\Delta_{w g}=\alpha-1 / 4$ and $\Delta_{c p}=3 / 4-\alpha$.

\section{Interaction and imitation between and within populations}

The first specificity of the two-population game analyzed here is that individuals in one population play against individuals of their own kind but also of the other population. We assume a unit mass population divided between Sanchos and Quixotes. The share of Sanchos in the overall population is represented by constant $s \in(0,1)$, and hence $1-s$ represents the ratio of Quixotes. Both types of players have the same set of two strategies: compliance and defection, $\{C, D\}$. Importantly, while they can switch between strategies, their preferences do not vary, and hence, they remain within their population. Thus, the set of social states in this two-population game can be written as, $X=\{\mathbf{x}=(p s,(1-p) s, q(1-s),(1-q)(1-s)): p, q \in[0,1]\}$, with $p$ (resp. $q$ ) the ratio of agents in the population of Sanchos (resp. Quixotes) who comply.

For each social state, $\mathbf{x}$, the payoff function for the two-population game can be computed as $F \mathbf{x}^{\prime}$ with,

$$
F=\left(\begin{array}{cccc}
1 & 1-d & 1 & 1-d \\
1+c-d & 0 & 1+c-d & 0 \\
1+\alpha \varepsilon & 1-d+\alpha \varepsilon & 1+\alpha \varepsilon & 1-d+\alpha \varepsilon \\
1+c-d-(1-\alpha) \varepsilon & -(1-\alpha) \varepsilon & 1+c-d-(1-\alpha) \varepsilon & -(1-\alpha) \varepsilon
\end{array}\right)
$$


For simplicity we will refer to the total population which complies (adding up Sanchos and Quixotes), denoted by $y=p s+q(1-s)$. Then the payoffs $F \mathbf{x}^{\prime}$ for the two populations can be rewritten as a function of $y:{ }^{8}$

$$
\pi(y)=\left(\begin{array}{c}
\pi_{\mathrm{c}}^{\mathrm{s}}(y) \\
\pi_{\mathrm{D}}^{\mathrm{s}}(y) \\
\pi_{\mathrm{c}}^{\mathrm{Q}}(y) \\
\pi_{\mathrm{D}}^{\mathrm{Q}}(y)
\end{array}\right)=\left(\begin{array}{c}
1-d+d y \\
y(1+c-d) \\
1-d+d y+\alpha \varepsilon \\
y(1+c-d)-(1-\alpha) \varepsilon
\end{array}\right) .
$$

From (6) it is clear that the expected payoff for Quixotes surpasses the expected payoff for Sanchos in the warm-glow, $\alpha \varepsilon$, while defection is less rewarding in the cold-prickle, $(1-\alpha) \varepsilon$.

$$
\pi_{\mathrm{C}}^{\mathrm{Q}}(y)=\pi_{\mathrm{c}}^{\mathrm{s}}(y)+\alpha \varepsilon, \quad \pi_{\mathrm{D}}^{\mathrm{Q}}(y)=\pi_{\mathrm{D}}^{\mathrm{s}}(y)-(1-\alpha) \varepsilon .
$$

As $\alpha$ approaches 1, the differences between Sanchos and Quixotes particularizes on the warm-glow from compliance, and the payoff to defection converges for the two populations. Conversely, when $\alpha$ tends to 0 , the gap between populations particularizes in the cold-prickle from non-compliance, while compliance is equally valued by the two type of agents.

Comparing the payoffs between strategies for the same population it follows:

$$
\pi_{\mathrm{D}}^{\mathrm{S}}(y)-\pi_{\mathrm{c}}^{\mathrm{s}}(y)=(d-1)+\sigma y>0, \quad \pi_{\mathrm{D}}^{\mathrm{Q}}(y)-\pi_{\mathrm{c}}^{\mathrm{Q}}(y)=\sigma(y-\Delta) .
$$

Within the population of Sanchos, defection always dominates compliance regardless of $y$. As for Quixotes, whether they prefer compliance or defection depends on how the share of compliance in the global population compares to $\Delta$. As shown in expression (3), the expression $\Delta$ (which represents the NE or the ESS if only a single population of Quixotes existed) takes values within the interval $(0,1)$, due to the snowdrift structure of the payoff matrix for Quixotes. These later would prefer to comply when few individuals comply, $y<\Delta$, and would prefer to defect in the opposite case.

\footnotetext{
${ }^{8}$ Superscripts S and Q refer to Sanchos and Quixotes, respectively. Subscripts C and D to compliance and defection.
} 
A population game defined exclusively for the population of Sanchos would be characterized by the convergence towards an evolutionary stable strategy of zero compliance. Similarly, for a population of Quixotes, the compliance rate at the equilibrium would be $\Delta$. Our main interest is to analyze the equilibrium for the two-population game proposed. With that aim, the imitation mechanism must be specified. And it is this mechanism which constitutes the second main feature of the proposed two-population game.

Considering an imitative revision protocol, the temporal evolution of the compliance rate in each population is determined by the share of non-compliance times the probability of switching to compliance, minus the share of compliance times the probability of switching to defection:

$$
\begin{aligned}
& \dot{p}=(1-p) \rho_{\mathrm{DC}}^{\mathrm{s}}-p \rho_{\mathrm{CD}}^{\mathrm{s}}, \\
& \dot{q}=(1-q) \rho_{\mathrm{DC}}^{\mathrm{Q}}-q \rho_{\mathrm{CD}}^{\mathrm{Q}},
\end{aligned}
$$

where $\rho_{i j}^{h}$ is the probability that an individual in population $h \in\{S, Q\}$ playing strategy $i \in\{C, D\}$ switches to the alternative strategy $j \in\{C, D\}, j \neq i$. This probability is determined by the likelihood that a revising agent is paired with an individual playing the alternative strategy, times the conditional imitation rate, $r_{i j}^{h}$. In the standard formulation of multi-population games (see, for example Sandholm 2010), when an individual in population $h$ receives a revision opportunity, she can only be paired with other individuals within her own population. Hence the likelihood of meeting someone playing the alternative strategy is given by the ratio of individuals in population $h$ playing this alternative strategy $j$. Thus, for Sanchos, the probability of switching from compliance to defection would read $\rho_{\mathrm{CD}}^{\mathrm{s}}=(1-p) r_{\mathrm{CD}}^{\mathrm{s}}$, and the mirror probability of switching from defection to compliance, $\rho_{\mathrm{DC}}^{\mathrm{s}}=p r_{\mathrm{DC}}^{\mathrm{s}}$ (similarly for Quixotes, changing $p$ by $q$, and $r_{i j}^{\mathrm{S}}$ by $r_{i j}^{\mathrm{Q}}$ ). Our proposal adds the possibility that she could also imitate the behavior of the agents belonging to the alternative population. Thus the probabilities of switching her strategy depend on the likelihood of being paired with someone in her own and also in the other population, who plays the alternative strategy. Hence, for Sanchos and Quixotes, this probabilities read: 
Compliance with social norms as an evolutionary stable equilibrium

$$
\begin{array}{ll}
\rho_{\mathrm{CD}}^{\mathrm{s}}=(1-p) s r_{\mathrm{CD}}^{\mathrm{SS}}+(1-q)(1-s) r_{\mathrm{CD}}^{\mathrm{SQ}}, & \rho_{\mathrm{DC}}^{\mathrm{S}}=p s r_{\mathrm{DC}}^{\mathrm{SS}}+q(1-s) r_{\mathrm{DC}}^{\mathrm{SQ}}, \\
\rho_{\mathrm{CD}}^{\mathrm{Q}}=(1-p) s r_{\mathrm{CD}}^{\mathrm{QS}}+(1-q)(1-s) r_{\mathrm{CD}}^{\mathrm{QQ}}, & \rho_{\mathrm{DC}}^{\mathrm{Q}}=p s r_{\mathrm{DC}}^{\mathrm{S}}+q(1-s) r_{\mathrm{DC}}^{\mathrm{QQ}},
\end{array}
$$

where $r_{i j}^{h k}$, with $h, k, \in\{S, Q\}$, and $i, j \in\{C, D\}$, represents the conditional imitation rate of an agent in population $h$ playing strategy $i$ who is paired with an agent in population $k$ playing strategy $j \neq i$.

Assuming a revision protocol governed by pairwise imitation, the conditional imitation rate is proportional to the gap between the payoffs of the randomly chosen opponent and the revising player. Thus, the conditional imitation rate of an $i$-player in population $h$ who meets a $j$-player in population $k$ reads:

$$
r_{i j}^{h k} \equiv r_{i j}^{h k}(y)=\left[\pi_{j}^{k}(y)-\pi_{i}^{h}(y)\right]_{+},
$$

where $[z]_{+}=z$ if $z>0$ and 0 otherwise. It is important to notice that imitation is only driven by the gap between payoffs, and individuals in one population will equally imitate agents from their same or the other population. ${ }^{9}$

A compliance individual, who can be a Sancho or a Quixote, can be paired with non-compliant Sanchos or non-compliant Quixotes. This gives a matrix of payoffs comparisons describing the conditional imitation rates from compliance to defection, $G_{\mathrm{CD}}(y)=\left(r_{\mathrm{CD}}^{h k}(y)\right)_{2 \times 2}$, with $h, k \in\{S, Q\}$. Likewise, the matrix $G_{\mathrm{DC}}(y)=$ $\left(r_{\mathrm{DC}}^{h k}(y)\right)_{2 \times 2}$ collects the conditional imitation rates from defection to compliance. ${ }^{10}$

$$
\begin{gathered}
G_{\mathrm{CD}}(y)=\left(\begin{array}{c}
{\left[\pi_{\mathrm{D}}^{\mathrm{s}}-\pi_{\mathrm{C}}^{\mathrm{s}}\right]_{+}\left[\pi_{\mathrm{D}}^{\mathrm{Q}}-\pi_{\mathrm{C}}^{\mathrm{s}}\right]_{+}} \\
{\left[\pi_{\mathrm{D}}^{\mathrm{s}}-\pi_{\mathrm{C}}^{\mathrm{Q}}\right]_{+}\left[\pi_{\mathrm{D}}^{\mathrm{Q}}-\pi_{\mathrm{C}}^{\mathrm{Q}}\right]_{+}}
\end{array}\right)=\sigma\left(\begin{array}{ll}
y+\frac{d-1}{\sigma} & {\left[y-\Delta_{c p}\right]_{+}} \\
{\left[y-\Delta_{w g}\right]_{+}} & {[y-\Delta]_{+}}
\end{array}\right) . \\
G_{\mathrm{DC}}(y)=\left(\begin{array}{l}
{\left[\pi_{\mathrm{C}}^{\mathrm{s}}-\pi_{\mathrm{D}}^{\mathrm{s}}\right]_{+}\left[\pi_{\mathrm{C}}^{\mathrm{Q}}-\pi_{\mathrm{D}}^{\mathrm{s}}\right]_{+}} \\
{\left[\pi_{\mathrm{C}}^{\mathrm{s}}-\pi_{\mathrm{D}}^{\mathrm{Q}}\right]_{+}\left[\pi_{\mathrm{C}}^{\mathrm{Q}}-\pi_{\mathrm{D}}^{\mathrm{Q}}\right]_{+}}
\end{array}\right)=\sigma\left(\begin{array}{ll}
0 & {\left[\Delta_{w g}-y\right]_{+}} \\
{\left[\Delta_{c p}-y\right]_{+}} & {[\Delta-y]_{+}}
\end{array}\right) .
\end{gathered}
$$

\footnotetext{
${ }^{9}$ One might introduce asymmetries, assuming that individuals are more willing to imitate their own kind rather than individuals belonging to the other population. We restrict to the symmetric case for simplicity of the exposition.

${ }^{10}$ The $y$ argument in the payoffs functions is removed when no confusion can arise.
} 
These matrices show the conditional imitation rates of a row individual belonging to a given population that meets a column individual from her or the other population playing the alternative strategy.

Fig. 2 Regions

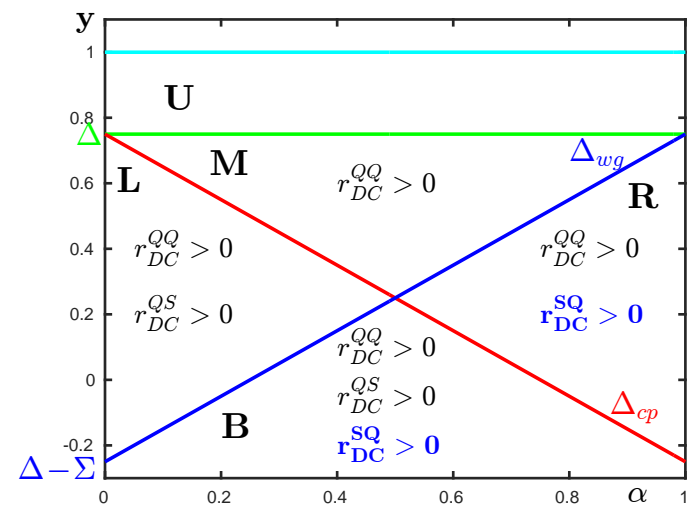

These matrices help distinguish five regions in the $\alpha-y$ plane (see Figure 2). The payoffs comparison is dependent on the share of the total population that complies, $y$, and on whether Quixotes attain great warm-glow from compliance ( $\alpha$ close to one), or strong cold-prickle from rules transgression ( $\alpha$ close to zero). The warmglow is relatively more important than the cold-prickle if $\alpha>1 / 2$, and vice versa.

From the prisoner's dilemma structure of the game for Sanchos, compliant Sanchos will always imitate non-compliant Sanchos and never the reverse: $r_{\mathrm{CD}}^{\mathrm{ss}}>0$ and $r_{\mathrm{DC}}^{\mathrm{ss}}=0$. As for the other matching pairs, Figure 2 displays five different regions, and highlights in each region when a non-compliant agent does worse than (and hence imitate), a compliant individual, either belonging to her own or the alternative population. ${ }^{11}$ From the snowdrift structure of the game for Quixotes, when the share of compliance in the global population is small, $y<\Delta$, non-compliance is highly detrimental for Quixotes who will be willing to imitate compliant Quixotes, $r_{\mathrm{DC}}^{\mathrm{QQ}}>0$. Conversely in region $\mathbf{U}, y>\Delta$ and the opposite occurs: $r_{\mathrm{CD}}^{\mathrm{QQ}}>0$.

If the warm-glow from compliance for Quixotes is strong $\left(\Delta_{w g}>0\right)$, and if $y<$ $\Delta_{w g}$, non-compliant Sanchos might find attractive to imitate compliant Quixotes,

\footnotetext{
${ }^{11}$ In the cases for which nothing is said, non-compliance provides a higher payoff.
} 
$r_{\mathrm{DC}}^{\mathrm{SQ}}>0$ (in regions $\mathbf{R}$ and $\mathbf{B}$ ). However, if $y>\Delta_{w g}$ then compliant Quixotes would imitate non-compliant Sanchos, $r_{\mathrm{CD}}^{\mathrm{SS}}>0$. On the other hand, if the cold-prickle from defection is strong, $\left(\Delta_{c p}>0\right)$ and compliance is small $y<\Delta_{c p}$, then non-compliant Quixotes would be inclined to imitate compliant Sanchos, $r_{\mathrm{DC}}^{\mathrm{OS}}>0$. Conversely, for $y>\Delta_{c p}$, compliant Sanchos would imitate non-compliant Quixotes, $r_{\mathrm{CD}}^{\mathrm{SQ}}>0$.

\section{Two asymptotically stable equilibria}

The evolutionary dynamics is characterized by the mean dynamics in (7)-(8), the probabilities of switching strategies in (9)-(10), and the conditional imitation rates defined in (12)-(13). For this evolutionary dynamics in variables $(p, q)$, presented in (26)-(27) in the Appendix, next proposition characterizes the different possible equilibria.

Proposition 1. The evolutionary dynamics in (26)-(27) presents two unstable equilibria, characterized by either full compliance, $\left(p^{*}, q^{*}\right)=(1,1)$, or zero compliance $\left(p^{*}, q^{*}\right)=(0,0)$. Moreover, under condition (2) there also exists a unique asymptotically stable fixed point of the evolutionary dynamics. This equilibrium can be of two types: ${ }^{12}$

1. Scenario $Q$ can be characterized by an upper bound on the warm-glow from compliance:

$$
w g \equiv \alpha \varepsilon \leq s(d-1)+(1-s)(c-d) \equiv \widehat{\alpha \varepsilon},
$$

or equivalently, in terms of the share of Sanchos in the overall population:

$$
s \leq 1-\frac{\alpha \varepsilon-(d-1)}{\sigma} \equiv 1-\Delta_{w g} \equiv \hat{s} .
$$

Under this condition, the equilibrium lies within regions $\mathbf{L}$ or $\mathbf{M}$, with

$$
p_{Q}^{*}=0, \quad q_{Q}^{*}=\frac{1+\Delta-\sqrt{(1-\Delta)^{2}+4(1-\alpha) s \varepsilon / \sigma}}{2(1-s)} \in(0,1) .
$$

2. The scenario $S Q$ is characterized by the opposite condition:

${ }^{12}$ Subscript $Q$ highlights that only Quixotes comply, while subscript SQ indicates that both Quixotes and Sanchos comply. 


$$
w g \equiv \alpha \varepsilon>s(d-1)+(1-s)(c-d) \equiv \widehat{\alpha \varepsilon} .
$$

or equivalently,

$$
s>1-\Delta_{w g} \equiv \hat{s} .
$$

The equilibrium lies within regions $\mathbf{R}$ or $\mathbf{B}$, with:

$$
p_{s Q}^{*}=\frac{-2 \sigma(1-s)-(d-1)+\sqrt{[d-1]^{2}+4 \sigma \alpha \varepsilon(1-s)}}{2 \sigma s} \in(0,1), \quad q_{s Q}^{*}=1 .
$$

Proof. See Appendix.

Under condition $(14),\left(0, q_{\mathrm{Q}}^{*}\right)$ is the unique asymptotically stable equilibrium, while under condition (17), the unique asymptotically stable equilibrium is given by $\left(p_{\mathrm{SQ}}^{*}, 1\right)$. The evolution of the share of compliance for Sanchos and Quixotes towards either of these two equilibria is depicted in Figures 3 and 4, for the parameters' values in (5). Additionally, Figure 3 considers $(s=0.5, \alpha=0.6)$ a lower ratio of Sanchos and a smaller warm-glow than Figure $4(s=0.8, \alpha=0.7)$.
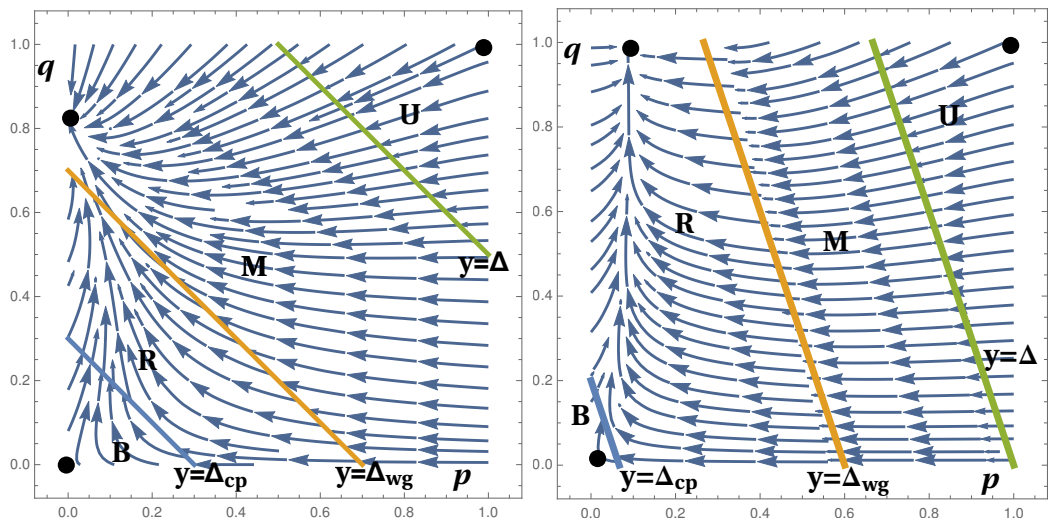

Fig. $3(p, q)$ for $s=0.5, \alpha=0.6$

Fig. $4(p, q)$ for $s=0.8, \alpha=0.7$

Under condition (17), compliant Quixotes do better than non-compliant Quixotes, and more importantly, also better than non-compliant Sanchos. And this remains true no matter how large the ratio of compliant Quixotes. Therefore, all Quixotes would end-up complying. When non-compliant Sanchos who revise their strategy are paired to compliant Quixotes, some switch to compliance, which leaves a pos- 
itive rate of compliance among formerly disobedient Sanchos, $p_{\mathrm{SQ}}>0$. The equilibrium in regions $\mathbf{B}$ or $\mathbf{R}$ is then characterized by a unitary compliance rate for Quixotes and a positive compliance rate for Sanchos (see Figure 4). Conversely, under condition (14), there is a threshold above which non-compliant Sanchos do better. Hence, as the number of compliant Quixotes increases, non-compliance becomes more attractive and ends up being the dominant strategy in the population of Sanchos.

Corollary 1. The threshold $\widehat{\alpha \varepsilon} \in(d-1, c-d)$ tends to $c-d$ when $s$ tends to 0 , and to $d-1$ when s tends to 1 . Therefore, if $\alpha \varepsilon<d-1<\widehat{\alpha \varepsilon}$, the equilibrium in scenario $Q$ is the only feasible equilibrium regardless of the value of $s$.

As Figure 5 shows, scenario $S Q$ involving compliance among Sanchos, requires Quixotes who attach a sufficiently large warm-glow to compliance, $w g>\widehat{\alpha \varepsilon}$. The greater the ratio of Sanchos in the overall population, $s$, the lower will be (as we will see later on) the share of compliance in the total population, $y$, and hence the stronger the reward that Quixotes obtain from compliance. Therefore, when paired with them, non-compliant Sanchos will imitate their compliant behavior. Conversely, if the ratio of Sanchos in the global population is very small, $s \rightarrow 0$, then the threshold $\widehat{\alpha \varepsilon}$ rises towards $c-d$. But since $\alpha \varepsilon \leq \varepsilon<c-d$ condition $w g>\widehat{\alpha \varepsilon}$ becomes highly demanding.

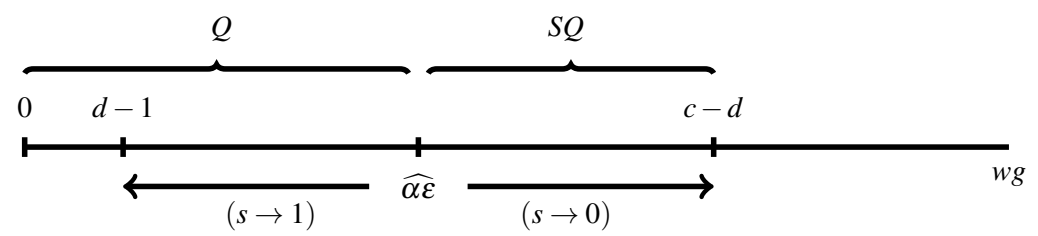

Fig. 5 Equilibrium type and warm-glow

According to this corollary, a solution $S Q$, with a positive compliance rate among Sanchos, is never feasible, if the warm-glow associated to compliance is not enough, by itself, to induce compliance among Quixotes $\left(\alpha \varepsilon<d-1\right.$, i.e. $\left.\Delta_{w g}<0\right)$. That is, if the willingness to comply for Quixotes when many other do not requires a strong cold-prickle from defection, then the payoff to compliant Quixotes is never 
large enough to induce imitation to non-compliant Sanchos. Therefore, Sanchos stick to their non-compliant dominant strategy. This is true regardless of how large the quantitative difference between Sanchos and Quixotes might be.

Corollary 2. A positive share of compliance among Quixotes requires:

$$
\varepsilon>\underline{\varepsilon}(s) \equiv \frac{d-1}{1-s(1-\alpha)}, \quad \text { with } \quad \underline{\varepsilon}(s) \in\left(d-1, \frac{d-1}{\alpha}\right), \underline{\varepsilon}^{\prime}(s) \leq 0 .
$$

Conditions in (2) are not enough to guarantee compliance in the two-population game. If the share of Sanchos in the overall population, $s$, tends to 0 , then $\underline{\varepsilon}$ tends to $d-1$ and the condition (20) coincides with the lower bound in (2). However, if $s \rightarrow 1$ condition (20) converges to the condition of a positive $\Delta_{w g}$, i.e. the warm-glow from compliance must be enough to induce compliance among Quixotes even with no cold-prickle from defection. Thus, $\Delta_{w g}>0$ is a sufficient condition for $q_{\mathrm{Q}}^{*}>0$, regardless of the value of $s$.

Next proposition presents the main features of the two equilibria described in Proposition 1 and depicted in Figures 3 and 4.

Proposition 2. The equilibrium under scenario $Q$ is characterized by $y_{Q}^{*}>\Delta_{w g}$, $p_{Q}^{*}=0$ and $q_{Q}^{*}$ given in (16) satisfying:

$$
q_{Q}^{*} \gtreqless \Delta \Leftrightarrow w g \equiv \alpha \varepsilon \gtreqless d-1+\sigma \Delta^{2}(1-s),
$$

or equivalently

$$
q_{Q}^{*} \gtreqless \Delta \Leftrightarrow s \gtreqless 1-\frac{\Delta_{w g}}{\Delta^{2}} .
$$

The equilibrium under scenario $S Q$ is characterized by $y_{S Q}^{*}<\Delta_{w g}, q_{S Q}^{*}=1$ and $p_{s Q}^{*} \in(0,1)$ given in $(19)$.

Proof. See Appendix.

To interpret this proposition in terms of the warm-glow, notice first that $d-1 \leq$ $d-1+\sigma \Delta^{2}(1-s) \leq \widehat{\alpha \varepsilon}$. As represented in Figure 6, if the warm-glow satisfies $w g \in\left(d-1, d-1+\sigma \Delta^{2}(1-s)\right)$, only Quixotes comply, and the share of compliance in this population remains below its equilibrium value in a single population of Quixotes, $\Delta$. A larger warm-glow, $w g \in\left(d-1+\sigma \Delta^{2}(1-s), \widehat{\alpha \varepsilon}\right)$, would push their compliance rate above $\Delta$, but Sanchos still refuse to comply. They will only 


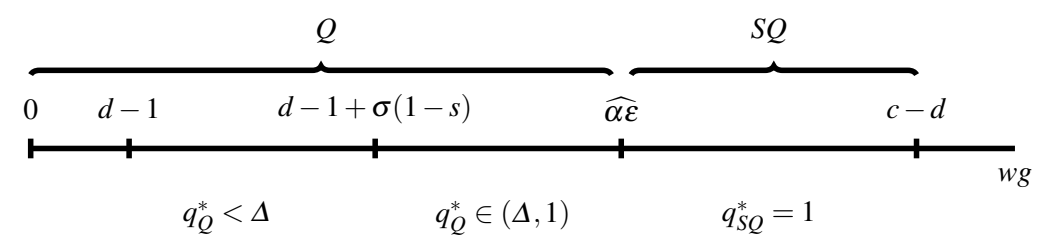

Fig. 6 Share of compliance among Quixotes and warm-glow

start to imitate Quixotes if the warm-glow that these latter attach to compliance is sufficiently strong, satisfying $w g>\widehat{\alpha \varepsilon}$.

Proposition 2 can also be read in terms of the ratio of Sanchos in the overall population, $s$, as shown in Figure 7. Assuming $\Delta_{w g}>0$ (otherwise, scenario $S Q$ is never feasible), then, since $\Delta \in(0,1)$, it is easy to see that:

$$
1-\frac{\Delta_{w g}}{\Delta^{2}}<1-\Delta_{w g}(\equiv \hat{s})<1
$$

If the ratio of Sanchos in the overall population is small, $s<\hat{s}$, only Quixotes comply. They will comply above the equilibrium in the single population game, $q_{\mathrm{Q}}^{*}>\Delta$, if $s \in\left(1-\Delta_{w g} / \Delta^{2}, \hat{s}\right)$, and they will comply below $\Delta$ if the ratio of Sancho is smaller $s<1-\Delta_{w g} / \Delta^{2}$. So, when the ratio of Quixotes is very large, they will be more strongly inclined to imitate the non-compliant behavior of Sanchos, showing a small share of compliance.

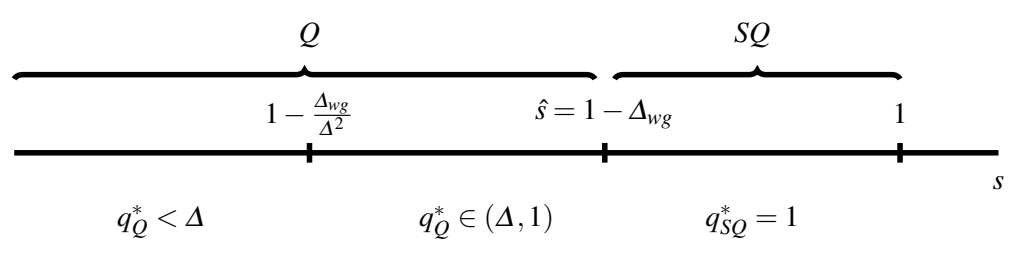

Fig. 7 Share of compliance among Quixotes and $s$

To better understand this dual possibility of over- and under-compliance, let us start with a single population of Quixotes at the equilibrium $q^{*}=\Delta$. The incorporation of some Sanchos within this population will have a twofold effect on the compliance decision of Quixotes. Firstly, a positive snowdrift effect is associated with the snowdrift nature of the game for Quixotes. Since we are in an equilibrium 
with zero compliance among Sanchos, the share of compliance in the global population immediately diminishes. Because each Quixote prefers compliance over universal disobedience, then a lower compliance rate increases the Quixote's incentive to comply. Secondly, a negative imitation effect is linked to the imitative revision protocol. With no Sanchos, compliant Quixotes only compare their payoff against the lower payoff of non-compliant Quixotes. However, with the entrance of some Sanchos who do not comply in this $Q$ scenario, compliant Quixotes can now be paired with non-compliant Sanchos who enjoy a larger payoff (in regions $\mathbf{L}$ and $\mathbf{M}$ in Figure 2). This negative imitation effect, given by $q(1-p) s\left[y-\Delta_{w g}\right]_{+}$, is stronger the greater the ratio of Sanchos, $s$, and it is weaker the stronger is the warm-glow from compliance.

In scenario $Q$, the share of compliance in the global population, $y_{Q}^{*}$, is at least as large as $\Delta_{w g}$ (according to Proposition 2, and also shown in Figure 3). This ratio, grows with the $w g$. However, even when Quixotes comply above $\Delta$, their overcompliance is not enough to counterbalance the zero compliance among Sanchos. The compliance rate in the global population is always lower than the compliance rate in a world without Sanchos: ${ }^{13} y_{\mathrm{Q}}^{*} \leq \Delta$.

In scenario $S Q$, the warm-glow surpasses $\widehat{\alpha \varepsilon}$, satisfying condition (17), then all Quixotes comply, and some Sanchos also imitate their compliant behavior. Now, although $\Delta_{w g}$ is larger, it serves as an upper bound for the share of compliance in the global population, $y_{\mathrm{SQ}}^{*} \leq \Delta_{w g} \leq \Delta$. Then, in this scenario, the share of compliance in the global population is again lower than its value in the case of a single population of Quixotes, $y_{\mathrm{SQ}}^{*} \leq \Delta$.

Propositions 1 and 2 suggest that large shares of compliance are associated with a strong warm-glow and a large ratio of Sanchos in the overall population. Next subsection analyzes whether this is a monotonous result. Does a higher warm-glow/a larger ratio of Sanchos increase compliance among Quixote and among Sanchos monotonously?

\footnotetext{
${ }^{13}$ This can be immediately proved from (16), provided that $y_{\mathrm{Q}}^{*}=q_{\mathrm{Q}}^{*}(1-s)$. Graphically, as shown in Figure 2, an equilibrium in region $\mathbf{L}$ or $\mathbf{M}$ always lies below the $y_{\mathrm{Q}}^{*}=\Delta$ line.
} 
Compliance with social norms as an evolutionary stable equilibrium

\section{Differences between populations and compliance shares}

This section analyzes how the share of compliance in each populations as well as the share of compliance in the global population are affected by the degree of dissimilarity between them. In particular we focus, on the one hand, on the gap that separates payoffs between the two populations, the warm-glow from compliance and the cold-prickle from defection experienced by Quixotes. On the other hand, we analyze how compliance is affected by the relative size of each population.

\subsection{The distance between Sanchos and Quixotes, $(\varepsilon, \alpha)$}

From condition (17) it follows that the feasibility of an equilibrium with positive compliance among Sanchos increases with the absolute distance between Sanchos

and Quixotes, $\varepsilon$, and particularly, with the warm-glow that Quixotes associate to compliance, rather than the cold-prickle to defection, measured by $\alpha$. Furthermore, as stated in the proposition below, the compliance rates for both types of agents and in both types of equilibria also increase.

Proposition 3. Under scenario $Q, p_{Q}^{*}=0$ and

$$
\frac{\partial q_{Q}^{*}}{\partial \alpha}, \frac{\partial q_{Q}^{*}}{\partial \varepsilon}>0 \Rightarrow\left(\frac{\partial y_{Q}^{*}}{\partial \alpha}=(1-s) \frac{\partial q_{Q}^{*}}{\partial \alpha}>0, \frac{\partial y_{Q}^{*}}{\partial \varepsilon}=(1-s) \frac{\partial q_{Q}^{*}}{\partial \varepsilon}>0\right), \quad \forall s \in(0,1) .
$$

Under scenario $S Q, q_{S Q}^{*}=1$ and

$$
\frac{\partial p_{s Q}^{*}}{\partial \alpha}, \frac{\partial p_{s Q}^{*}}{\partial \varepsilon}>0 \Rightarrow\left(\frac{\partial y_{s Q}^{*}}{\partial \alpha}=s \frac{\partial p_{s Q}^{*}}{\partial \alpha}>0, \frac{\partial y_{s Q}^{*}}{\partial \varepsilon}=s \frac{\partial p_{s Q}^{*}}{\partial \varepsilon}>0\right), \quad \forall s \in(0,1) .
$$

Proof. See Appendix.

At the equilibrium, the compliance rates for Sanchos (when they comply) and for Quixotes (when only they comply) increase with the discrepancy between the payoffs obtained by Sanchos and Quixotes, as measured by $\varepsilon$, and in particular, by the warm-glow from compliance for Quixotes, $\varepsilon \alpha$. Consequently, also the share of compliance in the global population rises with this discrepancy in payoffs between Sanchos and Quixotes. 

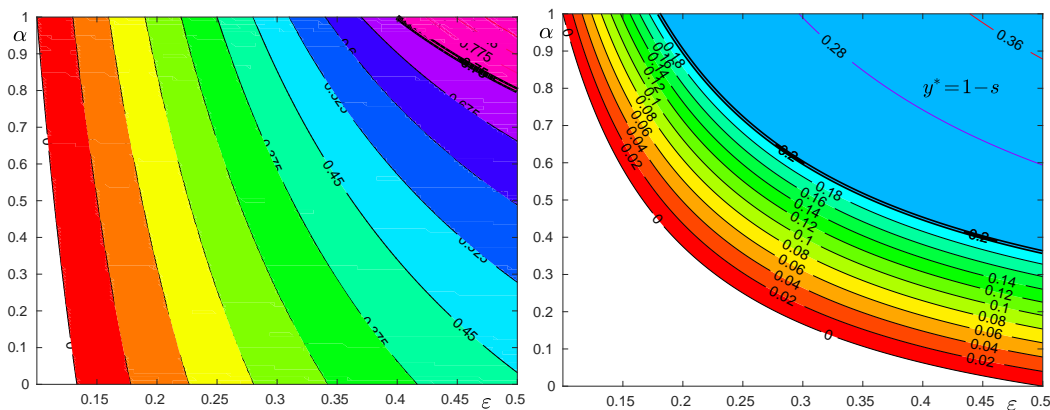

Fig. $8 y^{*}$ level curves for $s=0.25$ (left); and $s=0.8$ (right)

Figure 8 presents the level curves for the compliance rate in the global population in the $\varepsilon-\alpha$ plane for two different values $s=0.25$ and $s=0.8$. The level curve $y^{*}=$ $1-s$, is the frontier delimiting the two scenarios and represents the case in which all Quixotes comply, $q=1$ but still no Sancho imitates this behavior, $p=0$. In the gray region above this dotted line, the figure plots the level curves when all Quixotes comply together with some Sanchos, $y_{\mathrm{SQ}}^{*} \geq 1-s$; and below this dotted line, the level curves when only Quixotes comply, $y_{\mathrm{Q}}^{*} \in[0,1-s]$. The region below the curve $y^{*}=0$ represents $(\varepsilon, \alpha)$ combinations with null compliance among Sanchos and Quixotes.

This figure also highlights the result in Proposition 1, according to which the existence of an equilibrium with a positive compliance rate among Sanchos requires a sufficiently large gap between the payoffs attained by Sanchos and Quixotes. To reach this type of equilibrium, the less dissimilar the payoffs are ( $\varepsilon$ small), the more strongly the Quixotes must value compliance ( $\alpha$ large), and vice versa. If Quixotes are not too distinct from Sanchos, the latter would not imitate the former and only Quixotes would comply. In fact, if Quixotes attain roughly the same (but slightly higher) satisfaction from compliance, then it is the Quixotes who imitate Sanchos, ending up with a low, or even a zero compliance rate in the white region below the $y^{*}=0$ curve.

By comparing the two graphs in Figure 8, we observe three expected results. Firstly, the share of compliance in the global population decreases with the percentage of Sanchos in the global population. Any point in the $\varepsilon-\alpha$ plane is characterized by a greater share of compliance in Figure 8 (left) (with a lower ratio of Sanchos 
in the overall population). Secondly, the warm-glow required to induce compliance among Sanchos decreases with the ratio of Sanchos in the overall population. The gray area above the curve $y^{*}=1-s$ is greater for $s=0.8$ (right) than for $s=0.25$ (left). Finally, a greater ratio of Sanchos also makes it easier for some Quixotes to imitate the non-compliant behavior of Sanchos. Thus, a situation with null compliance in the overall population becomes more likely, represented by a greater white region below the level curve $y^{*}=0$. The effect of the ratio of Sanchos in the overall population is more deeply analyzed in the next subsection.

\subsection{The relative size of each population}

This section studies how the compliance rates in each population, as well as in the global population, are affected by the size of the population of Sanchos. ${ }^{14}$ It is worth recalling that we consider the size of the two populations as constant. Individuals may change their strategies, but their preferences remain unchanged, i.e. they remain within their population. We analyze how differences in populations' sizes affect, first the share of compliance in the global population, and second the shares of compliance in each population.

Proposition 4. The share of compliance in the global population decreases with the ratio of Sanchos in the overall population. This is true under scenario $Q$ and scenario $S Q$ :

$$
\frac{\partial y_{Q}^{*}}{\partial s}<0, \quad \frac{\partial y_{S Q}^{*}}{\partial s}<0 .
$$

Proof. Under scenario $Q$, the share of compliance in the global population is $y_{\mathrm{Q}}^{*}=$ $q_{\mathrm{Q}}^{*}(1-s)+0 s$, with $q_{\mathrm{Q}}^{*}$ given in (16). The derivative with respect to $s$ is unequivocally negative. Likewise, for scenario $S Q, y_{\mathrm{Q}}^{*}=1(1-s)+p_{\mathrm{SQ}}^{*} s$, with $p_{\mathrm{SQ}}^{*}$ given in (19). Again its derivative with respect to $s$ is unequivocally negative.

The share of compliance in the global population decreases with the relative size of the population of Sanchos. The reasoning is straightforward under scenario $Q$ :

\footnotetext{
${ }^{14}$ Because we have normalized the total population to 1, the ratio of Quixotes is just the complementary of the ratio of Sanchos.
} 
the larger the number of non-compliant individuals in the overall population, the lower the share of compliance. Under scenario $S Q$, some Sanchos comply meanwhile all Quixotes do comply. Hence, again, the greater the ratio of Sanchos, the lower must be the share of compliance in the global population. Although the ratio of Sanchos in the overall population has an undeniable discouraging effect in the share of compliance in the global population, its effect in each population is not so clear-cut.

Proposition 5. The share of compliance within the population of Quixotes under both scenarios can be expressed as a function of the ratio of Sanchos, s:

$$
q^{*}(s)=\left\{\begin{array}{cl}
\frac{1+\Delta-\sqrt{(1-\Delta)^{2}+4(1-\alpha) s \varepsilon / \sigma}}{2(1-s)} & \text { if } s \leq \hat{s}(w g \leq \widehat{\alpha \varepsilon}), \\
1 & \text { if } s>\hat{s}(w g>\widehat{\alpha \varepsilon}) .
\end{array}\right.
$$

This function satisfies $q_{Q}^{*}(0)=\Delta, q_{Q}^{*}(\hat{s})=1$, and it reaches its minimum at:

$$
\underline{s}_{q}=1+\sigma \frac{2 \Delta_{w g}-(1+\Delta) \sqrt{\Delta_{w g}}}{(1-\alpha) \varepsilon} .
$$

Thus, three situation are feasible:

1. $\alpha \varepsilon>d-1$, i.e. $\Delta_{w g}>0$ and, denoting by $\tilde{\alpha}=1-\Delta(1-\Delta) \sigma / \varepsilon$ :

a. $\alpha<\tilde{\alpha}$. Then $\underline{s}_{q} \in(0,1)$ and $\left(q^{*}\right)^{\prime}(s)<0$ for $s \in\left(0, \underline{s}_{q}\right)$ and $\left(q^{*}\right)^{\prime}(s)>0$ for $s \in\left(\underline{s}_{q}, \hat{s}\right)$.

b. $\alpha \geq \tilde{\alpha}$. Then $\underline{s}_{q} \leq 0$ and $\left(q^{*}\right)^{\prime}(s)>0$ for any $s \in(0, \hat{s})$.

2. $\alpha \varepsilon<d-1$, i.e. $\Delta_{w g}<0$. Then $\underline{s}_{q}$ is a complex number and $\left(q^{*}\right)^{\prime}(s)<0$ for all $s \in(0,1)$.

Proof. See Appendix.

As already mentioned, the relative size of the Sanchos' population has a twofold effect on the compliance decision of Quixotes: the snowdrift effect, which induces Quixotes to increase compliance in a world with a lower compliance rate, due to the enlargement of the portion of Sanchos; and the imitation effect, which induces Quixotes to imitate the non-compliant behavior of Sanchos.

If the warm-glow is enough to induce compliance when others disobey, $w g>$ $d-1$, then two situations are possible. For a relatively small $\alpha \in((d-1) / \varepsilon, \tilde{\alpha})$, 
Figure 9 shows that for an initially very small ratio of Sanchos the imitation effect is stronger. An increment in this population' size would reduce compliance among Quixotes who tend to imitate the non-compliant behavior of Sanchos. As the ratio of Sanchos rises the share of compliance decreases (because there are more Sanchos who do not comply, and because less Quixotes comply). In consequence, the snowdrift effect becomes stronger than the imitation effect and compliance start rising among Quixotes. Incidentally, when the ratio of Sanchos reaches $\hat{s}$, all Quixotes comply. Conversely, if the warm-glow is relatively strong wrt the coldprickle, $\alpha \geq \tilde{\alpha}$, then the snowdrift effect is stronger than the imitation effect even when the size of the Sanchos' population is very small. The share of compliance among Quixotes increases monotonously with the number of Sanchos in the overall population, up until every Quixote complies (see Figure 10).

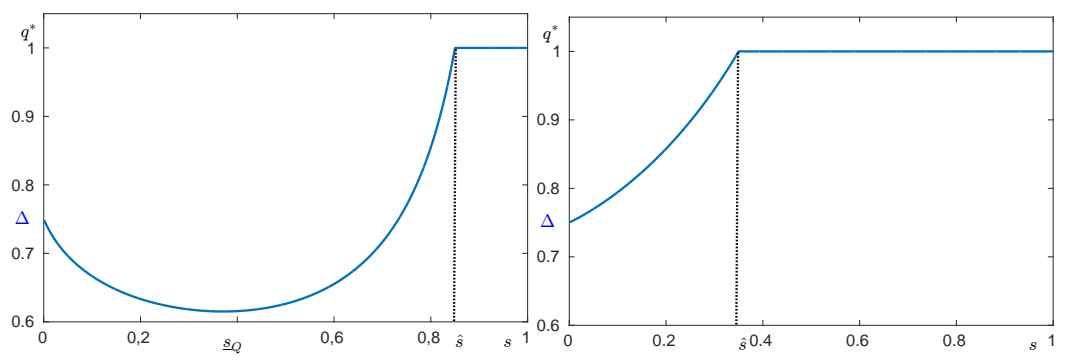

Fig. $9 q^{*}(s)$ for $\alpha=0.4<\tilde{\alpha}=0.81 \quad$ Fig. $10 q^{*}(s)$ for $\alpha=0.9>\tilde{\alpha}=0.81$

Fig. $11 q^{*}(s)$ for $\alpha=0.2$, with $\Delta_{w g}<0$

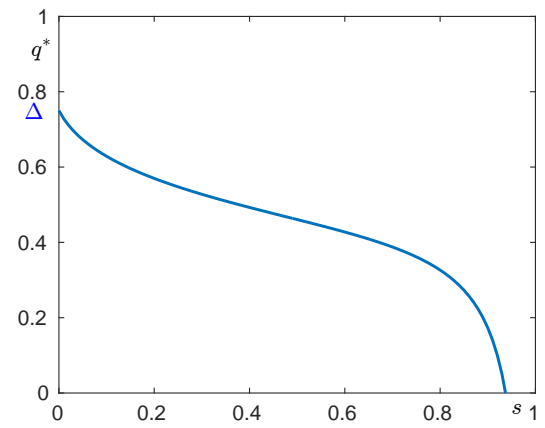

By contrast, if the warm-glow is not enough to guarantee compliance when others defect, then the share of compliance in the population of Quixotes decreases 
monotonously with the ratio of Sanchos. The imitation effect is stronger regardless of each population's size. More and more Quixotes imitate the non-compliant behavior of a larger population of non-compliant Sanchos, as shown in Figure 11.

Next proposition analyzes how the share of compliance in the population of Sanchos is affected by the relative size of this population.

Proposition 6. The share of compliance within the population of Sanchos in both scenarios can be defined as a function of the ratio of Sanchos:

$$
p^{*}(s)=\left\{\begin{array}{cc}
0 & \text { if } s \leq \hat{s}(w g \leq \widehat{\alpha \varepsilon}), \\
\frac{-2 \sigma(1-s)-(d-1)+\sqrt{[d-1]^{2}+4 \sigma \alpha \varepsilon(1-s)}}{2 \sigma s} & \text { if } s>\hat{s}(w g>\widehat{\alpha \varepsilon}) .
\end{array}\right.
$$

This function satisfies $p^{*}(\hat{s})=0$, and it reaches its maximum at:

$$
\bar{s}_{p}=\frac{-2 \sigma\left(1-\Delta_{w g}\right)+(c-d+\sigma) \sqrt{1-\Delta_{w g}}}{\alpha \varepsilon} .
$$

Proof. Considering $p_{\mathrm{SQ}}^{*}$ in (19) as a function of $s$, with the help of Mathematica, we compute the values at which $\left(p_{\mathrm{sQ}}^{*}\right)^{\prime}(s)=0$. This equation has a negative root and a positive root given by $\bar{s}_{p}$ in (25). From (19), we know that $p_{\mathrm{SQ}}^{*}(\hat{s})=p_{\mathrm{SQ}}^{*}(1)=0$, and $p_{\mathrm{SQ}}^{*}(s)$ is continuous and strictly positive within the interval $(\hat{s}, 1)$. Moreover there is a unique positive value $\bar{s}_{p}$ satisfying $\left(p_{\mathrm{SQ}}^{*}\right)^{\prime}\left(\bar{s}_{p}\right)=0$. In consequence, this value $\bar{s}_{p}$ must lie within the interval $\in(\hat{s}, 1)$ and $p_{\mathrm{SQ}}^{*}(s)$ must reach a maximum at this point.

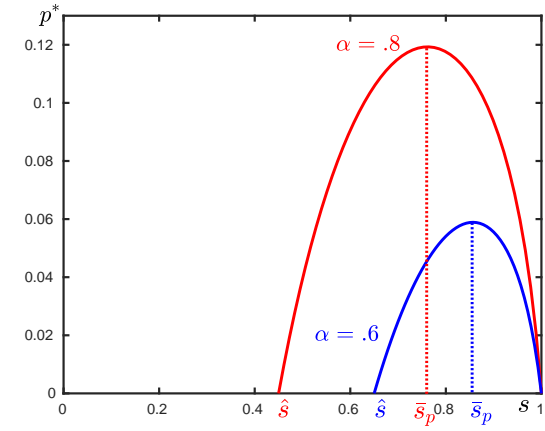

Fig. $12 p^{*}(s)$ for different $\alpha$

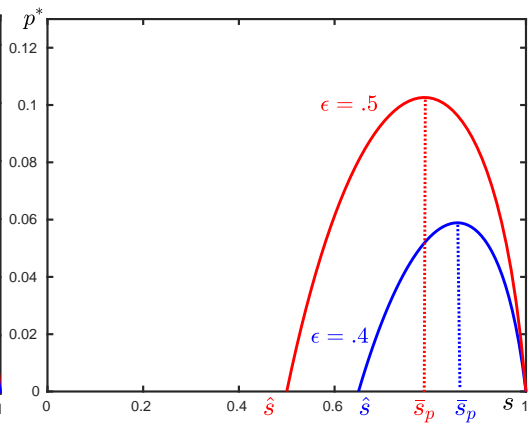

Fig. $13 p^{*}(s)$ for different $\varepsilon$ 
Compliance with social norms as an evolutionary stable equilibrium

As shown in Figure 12, the relative size of the Sanchos' population has a nonmonotonous effect on the compliance rate of these individuals. Again two forces are at stake here. As the number of Sanchos in the overall population grows, and the share of compliance in the global population decreases, also increases the reward to compliant Quixotes. In consequence more Sanchos are boosted to imitate their compliant behavior. This is the prevailing force up until $\bar{s}_{p}$. From this value on, a second and negative force dominates. As the number of Quixotes decays, although all of them comply, it becomes less and less likely for Sanchos to be paired with and to imitate their compliant behavior. Sanchos are more and more often paired to other Sanchos and the non-compliant dominant strategy in this population starts growing with $p_{\mathrm{SQ}}^{*}(s)$ tending to zero as $s$ goes to 1 .

Corollary 3. The value $\bar{s}_{p}$ decreases with the absolute distance which separates Sanchos from Quixotes and with the relative importance of the warm-glow with respect to the cold-prickle:

$$
\frac{\partial \bar{s}_{p}}{\partial \alpha}<0, \quad \frac{\partial \bar{s}_{p}}{\partial \varepsilon}<0
$$

for all $\alpha \in(0,1)$ and $\varepsilon$ satisfying condition (2).

The compliance rates increase with the absolute distance which separates Sanchos from Quixotes and with the relative importance of the warm-glow with respect to the cold-prickle:

$$
\frac{\partial p_{S Q}^{*}\left(\bar{s}_{p}\right)}{\partial \alpha}>0, \quad \frac{\partial p_{s Q}^{*}\left(\bar{s}_{p}\right)}{\partial \varepsilon}>0 .
$$

In particular, the maximum is higher.

Proof. See Appendix.

According to this corollary, the more dissimilar the two populations, or the more biased towards a greater warm-glow from compliance rather than a cold-prickle from defection, the wider is the interval with positive compliance among Sanchos. Moreover, the compliance rate among Sanchos, $p_{\mathrm{sQ}}^{*}(s)$, reaches its maximum for a lower ratio of Sanchos in the overall population. And this maximum is characterized by a larger compliance rate, as shown in Figures 12 and 13. 


\subsection{Real-life examples}

In our everyday life decisions, we continuously compare our gains with those of others implementing different options. We clearly do so when we take purchasing decisions as: whether to try a new restaurant instead of our favorite one, where to go on vacations, or whether to follow the fashion in order to look more attractive. Likewise, we also observe other people satisfaction in activities like paying taxes, contributing to voluntary associations, or recycling. We collect information from our friends and acquaintances and, more and more, we share experiences with unknown people through the use of the social networks and Internet. And we believe that it is seldom the case that we share their same preferences. Indeed although (for simplicity) we are assuming only two type of individuals, one could think that there are not just but many. Indeed, going to the extreme, each individual could have his specific payoff matrix, which could be very similar or far dissimilar to his new neighbor.

An example of how we imitate others and obtain different reward are marketing campaigns. The fancy car or the pair of jeans that we buy will rarely lead us to the success that actors pretend to enjoy in the commercials. More connected to social norms, an example could be the fund-raising television gala for charitable causes. We observe on TV how rewarding is donating for the celebrities, who act as role models or influencers. This induces some people, who would have not done otherwise, to collaborate. Marketing campaigns are often undertaken by the fiscal service on tax collection, or by the traffic authorities on road safety.

Examples of individuals having different preferences can be more clear when confronting people from clearly different cultures. An extreme example could be the missionaries, who are relatively few, whose preferences are rather different from the locals', and find highly rewarding to comply with their moral precepts. ${ }^{15}$ Thus, as Proposition 1 predicts, it is very likely that some of the locals embrace the missionaries' religion.

\footnotetext{
${ }^{15}$ Please, note that we do not mean any superiority of the missionaries' morality over other religions.
} 
To highlight the main findings of the paper, consider individuals who have grown up in a pleasant neighborhood with a well established culture of following social norms, either because neighbors really obtain satisfaction from compliance, or because they dislike or fear the penalty associated with defection. Assume that some of them move to a dirty and noisy neighborhood where people do not find it attractive to follow social rules. Then, some of the primitive neighbors might imitate the compliant behavior of the newcomers. This is more likely the more different the preferences of the new and the primitive neighbors are, and will only occur if these latter observe that the newcomers enjoy compliance, rather than dislike defection.

The compliance decisions of newcomers are influenced by two effects. Because of the deteriorated living environment, the snowdrift effect induces them to comply. Conversely, the inappropriate behavior of the primitive neighbors discourages compliance. These two effects are stronger the smaller is the share of newcomers. As stated in Proposition 5 and figures 9-11, if the new neighbors dislike defection, rather than enjoy compliance, the imitation effect is stronger and they will reduce their effort, the smaller their relative size. Conversely, if they attain a strong satisfaction from compliance, the snowdrift effect prevails and the lower their numbers, the more they will comply.

Conventional neighbors also value a clean and quiet environment, but they prefer others to pay the cost. As Proposition 6 and figures 12 and 13 state, if there are many newcomers, conventional neighbors will free ride on them and enjoy a better neighborhood. Conversely, if the number of newcomers is sufficiently small, the satisfaction of these latter is so large that some of the primitive neighbors will imitate them, aiming at a higher welfare. However, with even less newcomers, primitive neighbors seldom meet them, and therefore very few will imitate their compliant behavior.

Both newcomers and conventional neighbors will comply higher the more the newcomers' preferences differ from the primitive neighbors', and specially the greater their inner satisfaction from compliance. 


\section{Conclusions}

The paper analyzes the compliance with social norms as a social dilemma involving two types of individuals. The dilemma for pro-self Sanchos is described by a prisoner's dilemma game. On the other hand, pro-social Quixotes still have an incentive to free ride when others comply, but are willing to pay the cost of compliance when others deviate. For them, the social dilemma is described as a snowdrift game.

We have analyzed the compliance decisions of pro-self and pro-social normusing individuals, when their populations are not isolated from each other. Individuals in one population play against and imitate individuals from their own and from the other population. A two-population evolutionary game is defined involving Sanchos, with a payoffs matrix characteristic of a prisoner's dilemma game, and Quixotes, who obtain warm-glow from compliance and cold-prickle from defection, and whose payoffs matrix is characteristic from a snowdrift game.

Evolutionary dynamics is defined considering an imitative revision protocol, in particular, pairwise imitation. The imitative dynamics admits a unique asymptotically stable equilibrium. The nature of this stable equilibrium depends on the characteristics which describe the populations of Sanchos and Quixotes, as well as on the size of these populations. If the warm-glow that Quixotes attach to compliance is not too large, or equivalently if the ratio of Sanchos in the global population is low, then this equilibrium is characterized by zero compliance among Sanchos and a positive compliance rate (but not complete compliance) for Quixotes. Alternatively, if the warm-glow for Quixotes is high, then all Quixotes comply together with some Sanchos. What determines this latter equilibrium is a high degree of dissimilarity between Sanchos and Quixotes and particularly, a strong warm-glow from compliance rather than a strong cold-prickle from defection. Moreover, the size o the population of Sanchos also increases the likelihood that some Sanchos imitate the compliant behavior of Quixotes.

In the first equilibrium type, where only Quixotes comply, they can comply above or below their compliance in the case of a single population of Quixotes. Thus, at the equilibrium, they could over-comply to compensate the disobedient behavior of Sanchos, or imitate them and under-comply. Over-compliance would require a 
strong warm-glow from compliance or a large ratio of Sanchos in the overall population.

The absolute distance that separates Quixotes from Sanchos, or the relative size of the warm-glow over the cold-prickle, facilitates an equilibrium with a positive compliance rate among Sanchos. Moreover, the wider this gap, the higher the compliance rate in each population, and consequently in the global population. Conversely, the compliance rate in the global population decreases with the ratio of (reluctant-to-comply) Sanchos in the overall population. However, although compliance decreases globally with the percentage of Sanchos, its effect over the share of compliance in each population is diverse. In the equilibrium where only Quixotes comply, the ratio of Sanchos in the overall population may increase compliance if the warm-glow is large, or reduce compliance if it is small. With a moderate warmglow, the share of compliance for Quixotes is a u-shaped function of the ratio of Sanchos in the overall population. In the equilibrium with full compliance among Quixotes and partial compliance among Sanchos, a larger percentage of Sanchos increases their compliance rate initially. However, as this ratio becomes close to one the non-compliant strategy becomes dominant and the share of compliance among Sanchos decreases to zero.

Acknowledgements This study was funded by the Spanish Government (research projects ECO201452343-P and ECO2017-82227-P) and by the Regional Government of Castilla y León (research projects VA024P17 and VA105G18), co-financed by FEDER funds.

\section{Appendix: Proof of Propositions}

\section{Proof of Proposition 1}

The system dynamics reads:

$$
\begin{aligned}
& \frac{\dot{p}}{\sigma}=(1-p) q(1-s)\left[\Delta_{w g}-y\right]_{+} \\
& -p\left\{(1-p) s\left(y+\frac{d-1}{\sigma}\right)+(1-q)(1-s)\left[y-\Delta_{c p}\right]_{+}\right\}, \\
& \frac{\dot{q}}{\sigma}=(1-q)\left\{q(1-s)[\Delta-y]_{+}+p s\left[\Delta_{c p}-y\right]_{+}\right\} \\
& -q\left\{(1-q)(1-s)[y-\Delta]_{+}+(1-p) s\left[y-\Delta_{w g}\right]_{+}\right\} .
\end{aligned}
$$


From this system, the evolution of the compliance rates among Sanchos, $p$, and Quixotes, $q$, is analyzed separately for each of the five regions in the $\alpha-y$ plane, resumed in Figure 2. We can distinguish two situations depending on whether $\alpha<$ $1 / 2\left(\Delta_{w g}<\Delta_{c p}\right)$, or $\alpha>1 / 2\left(\Delta_{w g}>\Delta_{c p}\right)$. The possible equilibria in each region and their stability are also studied.

$\mathbf{U}: y>\Delta$. The dynamics reads:

$$
\begin{aligned}
& \dot{p}=-p\{(1-y)(y-\Delta) \sigma+[(1-p) s+(1-q)(1-s) \alpha] \varepsilon]\} \leq 0, \\
& \dot{q}=-q\{(1-y)(y-\Delta) \sigma+(1-p) s(1-\alpha) \varepsilon]\} \leq 0 .
\end{aligned}
$$

$\dot{p}<0$, except if $p=0$ or $p=q=1$, when $\dot{p}=0$. Similarly, $\dot{q}<0$, except for $q=0$ or $p=q=1$, when $\dot{q}=0$. The point $(0,0) \notin \mathbf{U}$, while $(1,1) \in \mathbf{U}$. Thus $(1,1)$ is the only equilibrium in this region and it is unstable.

M: $\max \left\{\Delta_{w g}, \Delta_{c p}\right\}<y \leq \Delta$. The dynamics in this region reads:

$$
\begin{aligned}
& \dot{p}=-p\left\{(1-p) s[(y-\Delta) \sigma+\varepsilon]+(1-q)(1-s)\left(y-\Delta_{c p}\right) \sigma\right\} \leq 0, \\
& \dot{q}=q\{(1-y)(\Delta-y) \sigma-(1-p) s(1-\alpha) \varepsilon\} .
\end{aligned}
$$

As in region $\mathbf{U}, \dot{p}<0$, except if $p=0$ or $p=q=1$, when $\dot{p}=0$, but $(1,1) \notin \mathbf{M}$.

Furthermore:

$$
\dot{q} \gtrless 0 \Leftrightarrow(1-y)(\Delta-y) \sigma \gtrless(1-\alpha)(1-p) s \varepsilon .
$$

From this dynamics, the only possible equilibrium in this region must satisfy $p=0$. In this situation $\dot{q}=0$ under equation:

$$
[1-q(1-s)][\Delta-q(1-s)]=(1-\alpha) s \frac{\varepsilon}{\sigma}
$$

Or equivalently, for $p=0, \dot{q} / q$ is given by the second order polynomial in $q$ :

$$
q^{2}(1-s)^{2}-q(1-s)(1+\Delta)+\Delta-(1-\alpha) s \frac{\varepsilon}{\sigma},
$$

which has one stable and one unstable root. The stable root is given by (16).

L: $\Delta_{w g}<y \leq \Delta_{c p} \leq \Delta(\alpha<1 / 2)$. The dynamics in this region reads: 


$$
\begin{aligned}
& \dot{p}=-p(1-p) s[y+d-1] \sigma \leq 0, \\
& \dot{q}=\{(1-q) y+q(1-p) s\}(\Delta-y) \sigma-s \varepsilon[\alpha(p-q)+(1-p) q)] .
\end{aligned}
$$

Still in this region $\dot{p}<0$, except if $p=0$ or $p=1$, when $\dot{p}=0$. For Quixotes:

$$
\dot{q} \gtrless 0 \Leftrightarrow\{(1-q) y+q(1-p) s\}(\Delta-y) \sigma \gtrless s \varepsilon[\alpha(p-q)+(1-p) q)] .
$$

The equilibrium in this region also requires $p=0$. Plugging this into the dynamics of $\dot{q}$ and equating to zero, leads again to equation (30).

The equilibrium $\left(0, q_{\mathrm{Q}}^{*}\right)$ may lie within this region $\mathbf{M}$ or region $\mathbf{L}$. It will be located in region $\mathbf{M}$ if $\max \left\{\Delta_{w g}, \Delta_{c p}\right\}<q_{\mathrm{Q}}^{*}(1-s) \leq \Delta$, and it will lie in region $\mathbf{L}$ if $\Delta_{w g}<q_{\mathrm{Q}}^{*}(1-s) \leq \Delta_{c p} \leq \Delta$.

$\mathbf{R}: \Delta_{c p}<y \leq \Delta_{w g} \leq \Delta(\alpha>1 / 2)$

$$
\begin{aligned}
& \dot{p}=\{p(1-y)+(1-s) q(1-p)\}(\Delta-y) \sigma-\varepsilon\{(1-p) y+(1-s) \alpha(p-q)\}, \\
& \dot{q}=(1-q) q(1-s)(\Delta-y) \sigma \geq 0 .
\end{aligned}
$$

In this region $\dot{q} \geq 0$, and $\dot{q}=0$ if $q=0$ (which does not belong to $\mathbf{R}$ ) or $q=1$. For Sanchos, $\dot{p} \gtrless 0$ if and only if:

$$
\left[y+(1-s) p \frac{1-q}{1-p}\right](\Delta-y) \sigma \gtrless \varepsilon\left[y+(1-s) \alpha \frac{p-q}{1-p}\right],
$$

In the limiting case of a very small ratio of compliant Sanchos, $\dot{p}$ can be approximated by:

$$
\left.\dot{p}\right|_{p=0}=(1-s) q \sigma\left[\Delta_{w g}-y\right],
$$

which in this region is positive, increasing in $\alpha$ and decreasing in $s$.

The equilibrium in this region requires $q=1$, and hence $y=p s+1-s$. Plugging this into the dynamics $\dot{p}$, it follows that $p$ must be either equal to 1 (but $p=q=1$ does not belong to $\mathbf{R}$ ) or satisfy equation:

$$
[p s+(1-s)](\Delta-p s-(1-s)) \sigma=\varepsilon[p s+(1-s)(1-\alpha)] .
$$

Equivalently, when $q=1$, the expression $\dot{p} /((1-p) \sigma)$ is given by the second order polynomial in $p$ :

$$
-p^{2} s^{2}-p s[2(1-s)+d-1]+(1-s)\left[\Delta_{w g}-(1-s)\right]
$$


which has one stable and one unstable root. The stable root is given by (19).

B: $y \leq \min \left\{\Delta_{w g}, \Delta_{c p}\right\} \leq \Delta$. The dynamics reads:

$$
\begin{aligned}
& \dot{p}=(1-p)\{(\Delta-y) y \sigma-\varepsilon[y-q(1-s) \alpha)]\}, \\
& \dot{q}=(1-q)\left\{p s \sigma\left(\Delta_{c p}-y\right)+q(1-s)(\Delta-y) \sigma\right\} \geq 0 .
\end{aligned}
$$

Since $y<\Delta_{c p}$ then $\dot{q} \geq 0$ except if $q=1$ or $p=q=0$, when $\dot{q}=0$. For Sanchos:

$$
\dot{p} \gtrless 0 \Leftrightarrow(\Delta-y) y \sigma \gtrless \varepsilon[y-q(1-s) \alpha] .
$$

This system has three equilibria. Two unstable equilibria $\left(p^{*}, q^{*}\right)=(0,0),\left(p^{*}, q^{*}\right)=$ $(1,1)$ (which does not belong to this region), and a stable equilibrium with $q=1$ and $p$ given by equation (31).

The equilibrium $\left(p_{\mathrm{SQ}}^{*}, 1\right)$ may belong to region $\mathbf{R}$ or region $\mathbf{B}$. It will lie in region $\mathbf{R}$ if $\Delta_{c p}<p_{\mathrm{sQ}}^{*} s+(1-s) \leq \Delta_{w g} \leq \Delta$, and it will lie in region $\mathbf{B}$ if $p_{\mathrm{SQ}}^{*} s+(1-s) \leq$ $\min \left\{\Delta_{c p}, \Delta_{w g}\right\} \leq \Delta$.

It is easy to see that $q_{\mathrm{Q}}^{*}>0$ for all $s \in(0,1)$. Moreover, under condition $s<$ $1-\Delta_{w g} \equiv \hat{s}$, it can be seen that $q_{\mathrm{Q}}^{*}<1$.

It is also immediate to see that condition $s<1-\Delta_{w g}$ implies $p_{\mathrm{SQ}}^{*}>0$. Likewise, $p_{\mathrm{sQ}}^{*}<1$ under condition $-\alpha \varepsilon s<c-d-\alpha \varepsilon$. But this inequality is always true under condition (2).

The proof of the asymptotic stability can be found in Cabo and García (2018).

\section{Proof of Proposition 2}

Conditions (21) and (22) are straightforward from the definition of $q_{\mathrm{Q}}^{*}$ in (16).

Under scenario $Q$, condition $y_{\mathrm{Q}}^{*}>\Delta_{w g}$ is equivalent to $q_{\mathrm{Q}}^{*}(1-s)>\Delta_{w g}$, or:

$$
\frac{1+\Delta-\sqrt{(1-\Delta)^{2}+4(1-\alpha) s \varepsilon / \sigma}}{2}>\Delta-(1-\alpha) \frac{\varepsilon}{\sigma},
$$

or equivalently:

$$
1-\Delta+2(1-\alpha) \frac{\varepsilon}{\sigma}>\sqrt{(1-\Delta)^{2}+4(1-\alpha) s \varepsilon / \sigma} .
$$

The LHS is positive, hence, if we square both sides, after some algebra, we end up with condition: 


$$
s<1-\Delta_{w g},
$$

which is equivalent to condition (14) that characterizes scenario $Q$.

Likewise, under scenario $S Q$, condition $y_{\mathrm{sQ}}^{*}<\Delta_{w g}$ is equivalent to $p_{\mathrm{sQ}}^{*} S+(1-s)<$ $\Delta_{w g}$. Following a similar analysis, we end up with condition $s>1-\Delta_{w g}$, which is equivalent to condition (17) that characterizes scenario $S Q$.

\section{Proof of Proposition 3}

In the scenario with $p_{\mathrm{Q}}^{*}=0$ and $q_{\mathrm{Q}}^{*}>0$,

$$
\frac{\partial q_{\mathrm{Q}}^{*}}{\partial \alpha}=\frac{s \varepsilon}{(1-s) \sigma \sqrt{(1-\Delta)^{2}+4(1-\alpha) s \varepsilon / \sigma}}>0,
$$

and

$$
\frac{\partial q_{\mathrm{Q}}^{*}}{\partial \varepsilon}=\frac{\sqrt{(1-\Delta)^{2}+4(1-\alpha) s \varepsilon / \sigma}+1-\Delta-2(1-\alpha) s}{2 \sigma(1-s) \sqrt{(1-\Delta)^{2}+4(1-\alpha) s \varepsilon / \sigma}} .
$$

This latter derivative is positive if and only if:

$$
\sqrt{(1-\Delta)^{2}+4(1-\alpha) s \varepsilon / \sigma}>2(1-\alpha) s-(1-\Delta) .
$$

If the RHS of this inequality was negative, $\partial q_{Q}^{*} / \partial \varepsilon>0$. Conversely, if the RHS was positive, raising both sides to the square, and rearranging terms,

$$
s \alpha>s-\left[1-\Delta+\frac{\varepsilon}{\sigma}\right] .
$$

But according to (15), an equilibrium with no compliance among Sanchos requires $s<1-\Delta+(1-\alpha) \varepsilon / \sigma<1-\Delta+\varepsilon / \sigma$. Therefore, the RHS in inequation above is negative and hence, it always holds, which proves $\partial q_{\mathrm{Q}}^{*} / \partial \varepsilon>0$.

In the scenario with $q_{\mathrm{SQ}}^{*}=1$ and $p_{\mathrm{SQ}}^{*}>0$,

$$
\frac{\partial p_{\mathrm{SQ}}^{*}}{\partial \alpha}=\frac{(1-s) \varepsilon}{s \sqrt{(\varepsilon-\sigma \Delta)^{2}+4 \sigma \alpha \varepsilon(1-s)}}>0, \quad \frac{\partial p_{\mathrm{SQ}}^{*}}{\partial \varepsilon}=\frac{(1-s) \alpha}{s \sqrt{(\varepsilon-\sigma \Delta)^{2}+4 \sigma \alpha \varepsilon(1-s)}}>0 .
$$

The marginal effect of $\alpha$ and $\varepsilon$ in the global compliance rates immediately follows, provided that $y_{\mathrm{Q}}^{*}=q_{\mathrm{Q}}^{*}(1-s)$ and $y_{\mathrm{SQ}}^{*}=p_{\mathrm{SQ}}^{*} s+(1-s)$.

\section{Proof of Proposition 5}


Considering $q_{\mathrm{Q}}^{*}$ in (16) as a function of $s$, one can prove that $\left(q_{\mathrm{Q}}^{*}\right)^{\prime}(s) \gtreqless 0$ if and only if:

$$
(1-\alpha)^{2}(1-s)^{2}\left(\frac{\varepsilon}{\sigma}\right)^{2}-\Delta_{w g}\left(1-\Delta^{2}+4(1-\alpha) \frac{\varepsilon}{\sigma}\right) \lesseqgtr 0 .
$$

It is ease to see that $\alpha \varepsilon<d-1$ implies that the expression in (32) is positive, and therefore, $\left(q_{\mathrm{Q}}^{*}\right)^{\prime}(s)<0$, regardless of the value of $s$.

For the more general case of $\alpha \varepsilon>d-1$, the expression in (32) vanishes for two values of $s$, one greater than one and the other given by $\underline{s}_{q}$ in (24). From Proposition 1 we know that $q_{\mathrm{Q}}^{*}<1$ for all $s \in(0, \hat{s})$ and $q_{\mathrm{Q}}^{*}(\hat{s})=1$.

- If $\underline{s}_{q} \in(0, \hat{s})$, then, being $q_{\mathrm{Q}}^{*}(s)$ a continuous function, it must hold true that $\left(q_{\mathrm{Q}}^{*}\right)^{\prime}(s)<0$ for $s \in\left(0, \underline{s}_{q}\right)$ and $\left(q_{\mathrm{Q}}^{*}\right)^{\prime}(s)>0$ for $s \in\left(\underline{s}_{q}, \hat{s}\right)$. Therefore, if $\underline{s}_{q} \in(0, \hat{s})$, $q_{\mathrm{Q}}^{*}(s)$ would start at $q_{\mathrm{Q}}^{*}(0)=\Delta$, decrease to reach its minimum at $q_{\mathrm{Q}}^{*}\left(\underline{s}_{q}\right)$ and increase from that point till $q_{\mathrm{Q}}^{*}(\hat{s})=1$.

- It is nonetheless possible that $\underline{s}_{q}<0$ and in that case, $q_{\mathrm{Q}}^{*}(s)$ would monotonously increase from $q_{\mathrm{Q}}^{*}(0)=\Delta$, for all $s \in(0, \hat{s})$, again to $q(\hat{s})=1$.

We can compute, with the help of Mathematica, the unique value of $\alpha$ at which $\underline{s}_{q}=0$, given by $\tilde{\alpha}=1-\Delta(1-\Delta) \sigma / \varepsilon$. Moreover, after some algebra, it can be proven that $\partial \underline{s}_{q} / \partial \alpha<0$ if and only if:

$$
(1+\Delta)^{2}(1-\alpha)^{2}\left(\frac{\varepsilon}{\sigma}\right)^{2}+4(1-\Delta)^{2} \Delta_{w g} \Delta>0 .
$$

And this is true whenever $\alpha \varepsilon>d-1$ and hence $\Delta_{w g}>0$. In consequence, if $\alpha>\tilde{\alpha}$, $\underline{s}_{q}<0$ and $q_{\mathrm{Q}}^{*}(s)$ increases for all $s \in(0, \hat{s})$, while for $\alpha<\tilde{\alpha}, \underline{s}_{q}>0$ and $q_{\mathrm{Q}}^{*}(s)$ shows a u-shape within $(0, \hat{s})$.

\section{Proof of Corollary 3}

The derivatives $\partial \bar{s}_{p} / \partial \alpha$ and $\partial \bar{s}_{p} / \partial \varepsilon$ are both negative under the same condition:

$$
[2(c-d)-\alpha \varepsilon](c-d+\sigma)>4(c-d) \sigma \sqrt{\left(1-\Delta_{w g}\right)} .
$$

After some tedious algebra, this condition can be transformed to:

$$
\left(1-\Delta_{w g}\right)\left[(c-d-\sigma)^{2}+2(c-d)\right]+4 \alpha \varepsilon(c-d)>0,
$$

which clearly holds. 
Proving that $\partial \hat{s} / \partial w g<0$ is straightforward.

Finally, as stated in Proposition 3, $\partial p_{\mathrm{SQ}}^{*} / \partial \alpha>0$ and $\partial p_{\mathrm{SQ}}^{*} / \partial \varepsilon>$. Therefore, also the maximum is reached at a higher value.

\section{References}

1. Alger I, Weibull JW (2013) Homo Moralis-preference evolution under incomplete information and assortative matching. Econometrica 81:2269-2302. doi:10.3982/ECTA10637

2. Andreoni J (1988) Why free ride? J Public Econ 37(3):291-304. doi:10.1016/00472727(88)90043-6

3. Andreoni J (1990) Impure Altruism and Donations To Public Goods: a Theory of Warm-Glow Giving. Econ J 100(401):464-477. doi:10.2307/2234133

4. Andreoni J (1995) Warm-Glow versus Cold-Prickle: The Effects of Positive and Negative Framing on Cooperation in Experiments. Q J Econ 110(1):1-21.

5. Breton M, Sbragia L, Zaccour G (2010) A Dynamic Model for International Environmental Agreements. Environ Resource Econ 45:25-48. doi: https://doi.org/10.1007/s10640-009-9304-6

6. Bontems P, Rotillon G (2000) Honesty in Environmental Compliance Games. Eur J Law Econ 10(1):31-41. doi:10.1023/A:1018786721348

7. Cabo, F. García-González, A. (2018) Inter-population interaction in a world of Quixotes and Sanchos. Mimeo.

8. de Young R (1996) Some Psychological Aspects of Reduced Consumption Behavior: The Role of Intrinsic Satisfaction and Competence Motivation. Environ Behav 28(3):358-409. doi:10.1177/0013916596283005

9. Doebeli M, Hauert C, Killingback T (2004) The Evolutionary Origin of Cooperators and Defectors. Science 5697: 859-862. doi: 10.1126/science.1101456

10. Grafton RQ, Kompas T, Long NV (2017) A brave new world? Kantian-Nashian interaction and the dynamics of global climate change mitigation. Eur Econ Rev 99: 31-42. doi: https://doi.org/10.1016/j.euroecorev.2017.04.002

11. Güth W (1995) An evolutionary approach to explaining cooperative behavior by reciprocal incentives. Int J Game Theory 24(4):323-344. doi:10.1007/BF01243036

12. Güth W, Yaari M (1992) Explaining reciprocal behavior in simple strategic games: An evolutionary approach. In: Witt U (ed) Explaining Process and Change: Approaches to Evolutionary Economics. University of Michigan Press, Ann Arbor, pp. 23-34

13. Miller JH, Andreoni J (1991) Can evolutionary dynamics explain free riding in experiments? Econ Lett 36(1):9-15. doi:10.1016/0165-1765(91)90047-O

14. Olson M (1971) The Logic of Collective Action : Public Goods and the Theory of Groups. Harvard University Press 
15. Ostrom E (2000) Collective Action and the Evolution of Social Norms. J Econ Perspect 14(3):137-158. doi:10.1257/jep.14.3.137

16. Sandholm WH (2010) Population Games and Evolutionary Dynamics. MIT Press, Cambridge 